\title{
Non alcoholic steatohepatitis
}

\section{Introduction}

Non-alcoholic fatty liver disease (NAFLD) encompasses a spectrum of disease ranging from simple steatosis to inflammatory steatohepatitis (NASH) with increasing levels of fibrosis and ultimately cirrhosis. NAFLD is closely associated with obesity and insulin resistance, and is now recognized to represent the hepatic manifestation of metabolic syndrome. ${ }^{1}$ Clinical importance of nonalcoholic fatty liver disease has grown in recent years, mainly in consequence of the obesity epidemics, sedentary habits and high calorie diet adopted by people of western countries, reflecting the increase in cardiovascular and endocrine metabolic diseases. It has been considered the most common liver disease and the most frequent cause of elevated aminotransferases and cryptogenic cirrhosis. ${ }^{2}$ Hepatocellular carcinoma is a well-recognized complication of NASH related cirrhosis. ${ }^{1}$ Several systems have been proposed for the histologic assessment of NAFLD, of which the Kleiner NAFLD activity score (NAS) is probably the most well established. ${ }^{3}$

The pathogenesis of NAFLD for long has been explained by the two hit theory. According to the traditional view, hepatic fat accumulation (i.e. pure fatty liver) is the first hit and the prerequisite for hepatocyte injury to develop, whereas cytokines, adipokines, bacterial endotoxin, mitochondrial dysfunction and/or endoplasmic reticulum stress represent the second hit for the progression to NASH. ${ }^{3,4}$ The traditional 'two-hit' pathophysiological theory has been challenged as knowledge of the interplay between insulin resistance, adipokines, adipose tissue inflammation and other less recognised pathogenetic factors have increased over the last decade. In particular, it has been suggested that hepatic steatosis may represent an epiphenomenon of several distinct injurious mechanisms rather than a true 'first hit'., ${ }^{4,5}$ For this reason, the initial 'two-hit' theory for explaining the progression from NAFLD to NASH is now being modified by the 'multiple parallel hits' hypothesis. In the 'multiple-hit' model, the first hit is insulin resistance and its associated metabolic disturbances. Hyperinsulinaemia, caused by insulin resistance, results in an increased hepatic de novo lipogenesis and impaired inhibition of adipose tissue lipolysis that ultimately leads to an increased efflux of free fatty acids from the adipose tissue to the liver. After the initial hepatic fat infiltration, the liver becomes extremely vulnerable to a series of hits that may follow, leading to hepatocyte injury and finally progressing from simple steatosis to NASH and fibrosis. Such multiple pathogenetic factors may include oxidative damage, dysregulated hepatocyte apoptosis, activation of the profibrogenic transforming growth factor (TGF)-beta pathway, dysregulation of multiple adipokines and hepatic stellate cell activation. All of these factors can act in complicated way to enhance the development and progression of the hepatic lesions through the NAFLD spectrum. ${ }^{4,6}$

The American Association for the Study of Liver Diseases has recently discussed the key endpoints and specific trial design issues that are germane for development of diagnostic biomarkers and treatment trials in patients with NASH. In terms of potential for progression, the recommendations suggest to classify patients within the NAFLD spectrum into two main categories, i.e. non - alcoholic steatohepatitis (NASH); and 'not steatohepatitis, with steatosis' (a category traditionally defined as 'pure fatty liver' or 'simple steatosis'). In general, only one-third of patients in the spectrum of NAFLD develop
Volume 2 Issue 3 - 2017

\author{
Shubha Lal \\ Department of Pathology, Sharda University, India
}

Correspondence: Shubha Lal, Department of Pathology, School of Medical Sciences \& Research, Sharda University, India, Tel+919671909783, Email shubha I504@gmail.com

Received: April 26, 2017| Published: May 10, 2017

NASH, which is histologically defined by the presence of lobular inflammation, portal inflammation, cellular ballooning and fibrosis. By contrast, 'not steatohepatitis, with steatosis' is characterised by simple fat infiltration or steatosis with minimal inflammation. ${ }^{4,7}$ For patients with early stage NASH, about $9 \%$ to $20 \%$ will progress to cirrhosis over a period of 5-10 years. In contrast, 'not steatohepatitis, with steatosis' tends to be stable over time. ${ }^{8,9}$

Imaging modalities commonly used in the diagnosis of NAFLD include ultrasound, computed tomography (CT) and magnetic resonance imaging (MRI The newer imaging modalities which are being used in diagnosis of NAFLD are MR elastography and ultrasound elastography. ${ }^{1,10}$ However, despite the availability of newer imaging modalities and serum markers for presumptive diagnosis of NAFLD, liver biopsy still remains the gold standard for definite diagnosis of non-alcoholic fatty liver disease. The most widely used histological grading and staging is the NAFLD activity score developed by Kleiner et al. ${ }^{11}$ The grading of the score is the sum of scores for various degrees of steatosis, lobular inflammation and hepatocellular ballooning. ${ }^{11}$ The quest for accurate tools to stage NAFLD related liver injury stems from a variety of recent developments. First the prevalence of NAFLD has grown to epidemic proportions; it is currently the most common cause of abnormal liver biochemistry and cryptogenic cirrhosis, and a frequent indication for liver transplantation. Second, it is now well recognized that NAFLD exists as a spectrum consisting of two major phenotypes (simple steatosis and steatohepatitis) that have drastically different clinical implications.,

\section{Acknowledgements}

None.

\section{Conflict of interest}

The author declares no conflict of interest.

\section{References}

1. Barsic N, Lerotic I, Duvnjak LS, et al. Overview and developments in noninvasive diagnosis of nonalcoholic fatty liver disease. World J Gastroenterol. 2012;18(30):3945-3954.

2. Dowman JK, Tomlinson JW, Newsome PN. Systematic review: the diagnosis and staging of non-alcoholic fatty liver disease and non-alcoholic steatohepatitis. Aliment Pharmacol Ther. 2011;33(5):525-540.

3. Levene AP, Goldin RD. The epidemiology, pathogenesis and histopathology of fatty liver disease. Histopathology. 2012;61(2):141-152. 
4. Yilmaz Y. Review article: is non alcoholic fatty liver disease a spectrum, or are steatosis and non alcoholicsteatohepatitis distinct conditions ? Aliment Pharmacol Ther. 2012;36(9):815-823.

5. Cortez Pinto H, De Moura M, Day C. Nonalcoholic steatohepatitis from cell biology to clinical practice. J Hepatol. 2006;44(1):197-208.

6. Tilg H, Moschen AR. Evolution of inflammation in non-alcoholic fatty liver disease: the multiple parallel hits hypothesis. Hepatology. 2010;52(5):1836-1846.

7. Sanyal AJ, Brunt EM, Kleiner DE, et al. Endpoints and clinical trial design for non-alcoholic steatohepatitis. Hepatology. 2011;54(1):344-353.

8. Harrison SA, Torguson S, Hayashi PH. The natural history of non alcoholic fatty liver disease: a clinical histopathological study. Am J Gastroenterol. 2003;98(9):2042-2047.
9. Adams LA, Lymp JF, St Sauver J, et al. The natural history of non alcoholic fatty liver disease: a population based cohort study. Gastroenterology. 2005;129(1):113-121.

10. Saadeh S, Younossi ZM, Remer EM, et al. The utility of radiological imaging in nonalcoholic fatty liver disease. Gastroenterology. 2002;123(3):745-750.

11. Kleiner DE, Brunt EM, Van Natta M, et al. Design and validation of a histological scoring system for nonalcoholic fatty liver disease. Hepatology. 2005;41(6):1313-1321. 